\title{
When UAV Meets IRS: Expanding Air-Ground Networks via Passive Reflection
}

\author{
Xiaowei Pang, Min Sheng, Senior Member, IEEE, Nan Zhao, Senior Member, IEEE, \\ Jie Tang, Senior Member, IEEE, Dusit Niyato, Fellow, IEEE, and Kai-Kit Wong, Fellow, IEEE
}

\begin{abstract}
Thanks to its flexibility and mobility, unmanned aerial vehicle (UAV) has been widely applied in wireless networks. However, UAV communications may suffer from blockage and eavesdropping in practical scenarios due to the complex environment. Taking the recent advances in intelligent reflecting surface (IRS) to reconfigure the propagation environments, in this article, we employ IRS to enhance the performance of UAVaided air-ground networks. First, we overview the combination of UAV and IRS, by introducing the diverse applications of IRS and the appealing advantages of UAV, and highlighting the benefits of combining them. Then, we investigate two case studies where the UAV trajectory, the transmit beamforming and the IRS passive beamforming are jointly optimized. In the first case study, by equipping the IRS on a UAV, the average achievable rate of the relaying network is maximized. In the second one, the IRS is deployed to assist the UAV-ground communication while combating the adversarial eavesdropper. Simulation results are provided to demonstrate the performance enhancement resulted from combining UAV and IRS in air-ground networks. Finally, we shed light on some challenging issues to be resolved for practical implementations in this direction.
\end{abstract}

Index Terms-Air-ground networks, beamforming design, intelligent reflecting surface, trajectory optimization, unmanned aerial vehicle.

\section{INTRODUCTION}

Unmanned aerial vehicles (UAVs) play a major role in the fifth-generation $(5 \mathrm{G})$ mobile networks and are also anticipated to provide high-speed and on-demand wireless connectivity for future networks [1]. Due to its distinctive channel characteristics, accurate channel modeling of the UAV-aided air-ground communication is of pivotal importance to the performance analysis and system design for the networks [2]. Due to flexibility in deployment and its cost-effectiveness, UAVs can be employed to assist the ground base station (BS)

X. Pang and N. Zhao (Corresponding Author) are with the School of Information and Communication Engineering, Dalian University of Technology, Dalian 116024, China. (e-mail: xiaoweipang00@mail.dlut.edu.cn, zhaonan@dlut.edu.cn).

M. Sheng is with the State Key Laboratory of Integrated Services Networks, Xidian University, Xi'an 710071, China (e-mail: msheng@mail.xidian.edu.cn).

Jie Tang is with the School of Electronic and Information Engineering, South China University of Technology, Guangzhou 510641, China (e-mail eejtang@scut.edu.cn).

D. Niyato is with the School of Computer Science and Engineering, Nanyang Technological University, Singapore 639798 (e-mail: dniyato@ntu.edu.sg).

K.-K. Wong is with the Department of Electronic and Electrical Engineering, University College London, London WC1E 6BT, U.K. (e-mail: kaikit.wong@ucl.ac.uk).

This research was supported in part by the National Key R\&D Program of China under Grant 2020YFB1807002, and the National Natural Science Foundation of China (NSFC) under Grant 61871065. (Corresponding author: Nan Zhao.) to serve users of high data traffic and overloaded cases [1]. Moreover, UAVs can be combined with the key technologies at the physical layer to achieve higher capacity, such as non-orthogonal multiple access (NOMA), millimeter wave (mmWave), and so forth. For example, the uplink transmission in a cellular network was optimized in [3], where the UAV uses NOMA to transmit signals to its connected BSs, sharing the spectrum with ground users. In addition, UAVs can be utilized as friendly jammers to improve the security of wireless networks, as revealed in [4], where the secrecy performance of ground communications was investigated in the presence of a UAV jammer.

On the other hand, with massive low-cost passive reflecting elements, intelligent reflecting surface (IRS) can modify the amplitude and phase of the incident signals, and becomes a promising technique to reconfigure the propagation environments and improve network performance [5]. Compared with existing techniques, such as active relay and backscattering communication, IRS requires much less energy consumption and can be deployed easily on building facades, ceilings and walls. Recently, IRS has been considered in terrestrial networks to enhance capacity [6], energy efficiency [7], and physical layer security [8]. Specifically, the phase shifts of reflecting elements can be jointly optimized with the transmission control of transceivers in diverse network designs to achieve various communication goals.

Despite the advantages of UAV communication, the air-toground channels may suffer from blockage due to the complex terrain and surroundings. Moreover, the information security of legitimate users may not be guaranteed in the presence of eavesdropping. To tackle these issues, IRS can be employed in UAV-aided air-ground networks to obtain a favorable propagation environment and improve the communication quality of desired users. Meanwhile, IRS can also cancel out the undesired signals to suppress the interference and prevent adversarial eavesdropping by properly designing the passive beamforming. Recently, there are emerging studies combining UAV and IRS [9]-[15] to improve the performance of airground networks. Specifically, the coverage of UAV can be expanded with the help of IRS, and thus various quality of service (QoS) requirements of users can be supported. Compared to being installed on a fixed building, IRS enjoys more deployment flexibility and a wider range of signal reflection when mounted on a mobile UAV. In this article, we focus on combining UAV and IRS to enhance the performance of airground networks, with both terrestrial and aerial IRSs considered. First, we highlight the appealing benefits of combining UAV and IRS. Then, to take advantages of UAV mobility, IRS 


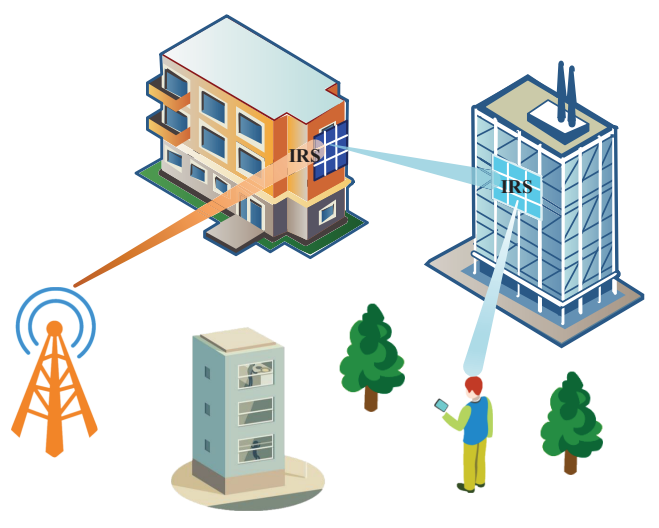

(a) IRS-enhanced terrestrial communication

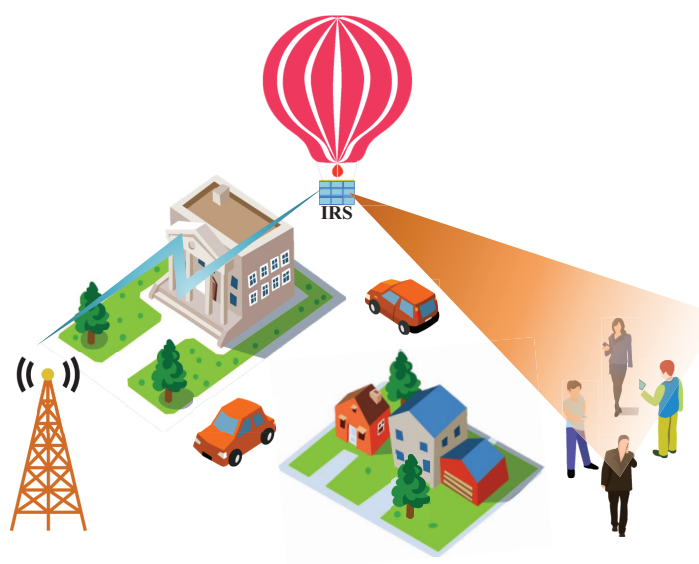

(c) Aerial IRS assisted relaying networks

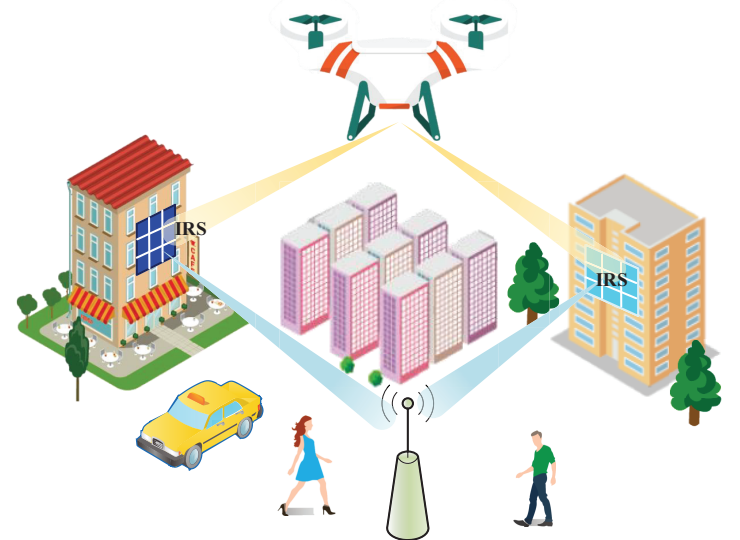

(b) IRS-assisted aerial communication

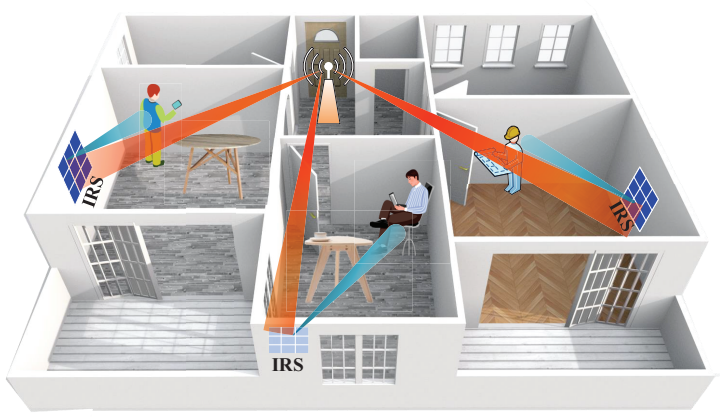

(d) IRS-aided indoor communication

Fig. 1. Typical applications of IRS in wireless networks.

passive reflecting and multi-antenna beamforming, we jointly optimize them for air-ground networks in two case studies. In the first case, the IRS is mounted on a UAV to enhance the communication quality from the BS to the user. In the second one, the IRS is utilized to assist the transmission from the UAV to the ground. Some interesting research challenges are also discussed.

The remainder of this article is organized as follows. In the next section, the complementary features of UAV and IRS are presented. Then, by equipping UAV with IRS, we jointly design the UAV trajectory, and the active and passive beamforming to maximize the average achievable rate. Furthermore, we investigate the IRS-assisted UAV networks to facilitate the UAV-ground communication and reduce information leakage. Finally, open research challenges are discussed, followed by our conclusions.

\section{Potentials of Integrating UAV And IRS}

In this section, we first introduce the distinctive characteristics of IRS and UAV, and then discuss the potential benefits of UAV and IRS integration.

\section{A. Architecture and Typical Applications of IRS}

IRS is a man-made two-dimensional metasurface, which consists of an array of passive scattering elements and can be controlled in a software-defined manner. Specifically, fieldprogrammable gate array can be applied as the controller, which serves as a gateway to communicate with external devices and distribute corresponding control decisions to each element. Receiving the control information via wired or wireless links, each element can induce an amplitude and/or phase change to the impinging electromagnetic wave. As a result, through the joint phase control of all reflecting elements, the direction and strength of the reflected wave can be changed.

In contrast to the active relay that assists communication by signal regeneration and retransmission, IRS only passively reflects the received signals without requiring any transmit radio-frequency (RF) chains, and thereby is much more energy efficient. Moreover, IRS can reflect the same message as that of the direct path and has the potential to enhance the desired signal or suppress the interference. Thus, IRS is envisioned to revolutionize future networks, and its typical applications are illustrated in Fig. 1. Thanks to the easy installation and conformal geometry, IRSs can be mounted on the facade of buildings to enhance the terrestrial or aerial communications. Another application considers that IRS can be carried by an aerial platform to help bypass obstacles and create virtual lineof-sight (LoS) links between the BS and its serving users. Furthermore, when some users are located in the dead zones where the direct receiving links are blocked, IRSs can be mounted indoors to help reflect signals for extending the range. 
TABLE I

SUMMARY OF THE RESEARCH COMBINING UAV AND IRS

\begin{tabular}{|c|c|c|c|c|}
\hline Reference & Objective & Optimization variables & Installation of IRSs & Mobility of UAV \\
\hline [9] & $\begin{array}{l}\text { Maximizing the average } \\
\text { achievable rate }\end{array}$ & $\begin{array}{l}\text { UAV trajectory and IRS passive beam- } \\
\text { forming }\end{array}$ & Mounted on a building & Mobile \\
\hline [10] & $\begin{array}{l}\text { Maximizing the received } \\
\text { power }\end{array}$ & $\begin{array}{l}\text { Trajectory, beamforming at UAV, and } \\
\text { passive beamforming at IRSs }\end{array}$ & Mounted on buildings & Mobile \\
\hline$[11]$ & $\begin{array}{l}\text { Maximizing the IRS data } \\
\text { transmission }\end{array}$ & $\begin{array}{l}\text { UAV trajectory, IRS phase shift, and } \\
\text { IRS scheduling }\end{array}$ & Mounted on buildings & Mobile \\
\hline$[12]$ & $\begin{array}{l}\text { Maximizing the secrecy } \\
\text { rate }\end{array}$ & $\begin{array}{l}\text { Trajectory, power control of UAV, and } \\
\text { IRS phase shift }\end{array}$ & Mounted on buildings & Mobile \\
\hline [13] & $\begin{array}{l}\text { Maximizing the rate of the } \\
\text { strong user }\end{array}$ & $\begin{array}{l}\text { Location, beamforming and phase shift } \\
\text { of IRS-UAV }\end{array}$ & Equipped at a UAV & Static \\
\hline [14] & $\begin{array}{l}\text { Maximizing the transmis- } \\
\text { sion capacity }\end{array}$ & $\begin{array}{l}\text { Location and reflection parameters of } \\
\text { IRS-UAV }\end{array}$ & Equipped at a UAV & Static \\
\hline [15] & $\begin{array}{l}\text { Maximizing the total ener- } \\
\text { gy efficiency }\end{array}$ & BS beamforming and IRS phase shift & Equipped at a UAV & Static \\
\hline
\end{tabular}

\section{B. Advantages of UAV Communication}

UAVs have been applied in a wide range of tasks, including cargo delivery, weather monitoring, emergency communication and so on. In particular, UAV-aided air-ground communication is expected to enhance coverage and provide significant flexibility for future wireless networks. The promising advantages of UAV communication can be summarized as follows.

LoS Links: Compared to terrestrial communication, UAVs have a higher probability to establish LoS air-ground communication links, and thus are more likely to have better wireless channels. In particular, short-distance LoS links can be established by carefully choosing the hovering location or the trajectory of UAV to enhance the quality of communication links.

Dynamic Deployment: The high mobility of UAVs offers new opportunities for performance improvement and adaptive communication in air-ground networks. For the deployment of a static UAV, we can find the optimal position to extend the coverage. With the mobility fully exploited, the UAV trajectory can be dynamically optimized according to user positions and the surrounding environment to satisfy the QoS requirements.

\section{Joint Design of UAV and IRS for Air-Ground Networks}

Due to the appealing advantages of UAV and IRS, their integration can significantly boost the network performance. However, the research on the joint utilization of UAV and IRS in air-ground networks is still in an early stage. Some existing works that present the joint design of UAV and IRS are outlined in Table I. Specifically, the mobility of UAV is fully exploited in [9]-[12], where the trajectory of UAV and the passive beamforming at IRS are jointly optimized in IRSassisted air-ground networks. In contrast, [13]-[15] consider a static UAV equipped with an IRS, and the reflecting phase shifts are optimized. In particular, the horizontal location of IRS-UAV is also optimized in [13] and [14]. The main benefits and challenges of combining UAV and IRS can be summarized as follows.

- Virtual LoS links can be built up between UAV and ground nodes via IRS in air-ground networks when there are no direct LoS links due to blockage. Thus, the communication quality from the UAV to the users can be enhanced, which is beneficial to coverage extension.

- When carried by UAV, the location of IRS can be intelligently and dynamically adjusted along with the UAV's movement. Compared with terrestrial IRSs that are mounted on fixed surfaces, the mobility of UAV provides a new degree of freedom for IRS design.

- Being mounted on a UAV, the IRS is more likely to establish LoS links with both the transmitter and receiver. Compared to the terrestrial IRS that can only help the transmission between two nodes located in half of the space, equipping IRS on UAV is able to achieve the fullangle reflection and expand the wireless coverage.

- IRS and UAV can also work in the mmWave or terahertz (THz) band, offering a ultra-wide bandwidth to fulfil the high data rate requirement. However, the mmWave and $\mathrm{THz}$ links face critical issues of blockages and severe path loss, which can be effectively compensated via the reconfigurability of IRS and the mobility of UAV.

- Despite the advantages above, IRS-UAV faces several new challenges such as the endurance and controllability of UAV when carrying IRS, the size, orientation and placement of IRS, as well as the difficulty in channel estimation. For the effective IRS-UAV deployment, the IRS's and UAV's practical constraints need to be taken into account together with the transmission design.

Despite of the abovementioned works, the trajectory of a flying IRS equipped at the UAV has not been well designed in air-ground networks. With IRS equipped at the UAV, the reflecting phase shifts will change with the mobility of UAV. Moreover, blockage and eavesdropping are challenging issues for air-ground channels, and deploying IRS is a promising solution to these problems by reconfiguring the propagation environment. Focusing on these two directions, in the following sections, two case studies will be investigated by jointly optimizing the UAV trajectory, the transmit beamforming and the phase shift of IRS. In the first scheme, IRS is equipped at the UAV to maximize the average achievable rate between two terrestrial nodes through joint optimization. Furthermore, the 


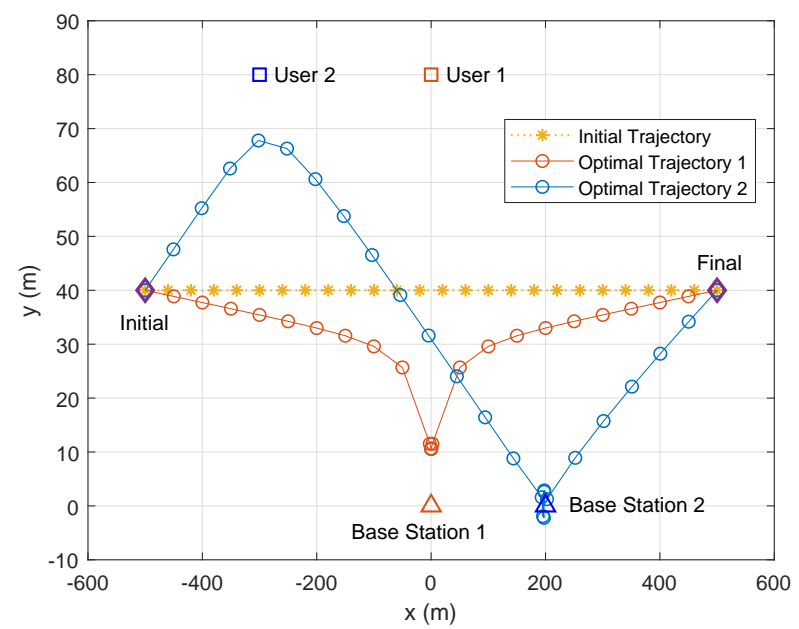

(a)

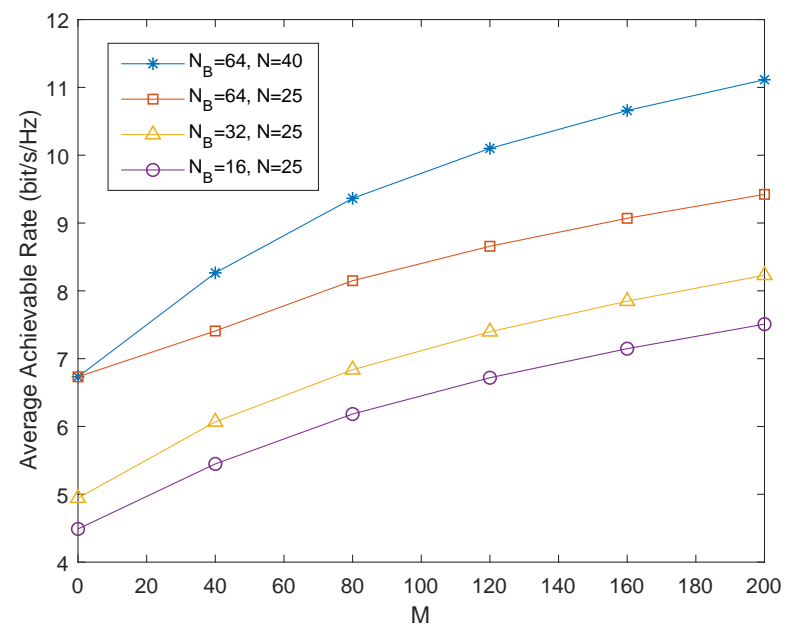

(b)

Fig. 2. (a) The optimized trajectories of UAV in two topologies; (b) The average achievable rate versus the number of IRS reflecting elements with different number of transmit antennas and time slots.

joint trajectory and beamforming design is presented for IRSassisted air-ground networks with eavesdropping considered.

\section{ENHANCING AIR-GRound COMMUNiCATION VIA IRS-EQUIPPED UAV}

In this section, we consider a terrestrial communication network enhanced by a UAV equipped with an IRS, which can be referred to Fig. 1(c). Since the direct link between the BS and its served user experiences severe path loss and blockages, the UAV equipped with a number of reflecting elements is employed to establish two connected LoS links between them. By leveraging the mobility of UAV, the flying trajectory of IRS should be adjusted according to the environment, and is jointly optimized with the phase shift to enhance the communication quality.

\section{A. Joint Trajectory and Beamforming Optimization}

In the UAV relaying network enhanced by IRS, we assume that the IRS mounted on the UAV consists of $M$ reflecting elements, the BS is equipped with $N_{B}$ antennas, and the user has a single antenna. The IRS-UAV is assumed to fly at a fixed altitude $H$ with the flying period $T$ and the maximum speed $V_{m}$. For ease of trajectory design, $T$ is further divided into $N$ small time slots so that the location of IRS-UAV in each time slot can be considered approximately unchanged. Thus, the horizontal distance that the IRS-UAV moves during each time slot is constrained to be smaller than $V_{m} T / N$. The initial and final locations of UAV during the flight are predefined in the network. Utilizing multiple antennas at the BS, the transmit beamforming vector in each time slot can be carefully designed. Moreover, the phase-shift matrix of IRS-UAV is a diagonal matrix, each diagonal entry of which indicates the phase shift incurred by the corresponding reflecting element. When modeling the channel between the BS and the user, we adopt a Rayleigh fading model considering multi-path propagation caused by rich scattering environment. In contrast, the air-ground channels of BS-to-UAV and UAV-to-user are assumed to be LoS links. It's worth pointing out that the orientation of IRS mounted on UAV can affect the air-ground channels, and herein we assume a reflecting surface along the horizontal plane. All the channel state information (CSI) is assumed to be available by employing existing channel estimation techniques.

Specifically, we aim at jointly optimizing the UAV trajectory, the passive beamforming at the IRS and the transmit beamforming at the BS, to maximize the average achievable rate at the user over $N$ time slots, subject to the transmit power, the UAV's practical mobility and the IRS's phaseshift constraints. However, the joint optimization problem is non-convex and intractable, which can be divided into three subproblems to solve. Furthermore, an iterative algorithm is presented to obtain the suboptimal solution by alternately solving these three subproblems until convergence is reached. The first subproblem is to obtain the active beamforming vector, which can be solved based on the principle of maximum ratio transmission. Then, the closed-form expression of phase shifts can be derived to maximize the receiving signal power in each time slot. As for the UAV trajectory optimization subproblem, it is non-convex. Therefore, the successive convex approximation (SCA) and first-order Taylor approximations are applied to obtain its suboptimal solution. It's worth noting that the optimal active and passive beamforming can be directly obtained in each iteration and the user can enjoy the most benefit of the channel gains from both the BS and the IRS-UAV. The objective value of the trajectory optimization subproblem usually serves as a lower bound of the original one due to the non-convexity. Since the achievable rate is nondecreasing over iterations, at least a locally optimal solution can be obtained by the algorithm.

\section{B. Numerical Results}

To evaluate the performance of the proposed scheme, we perform simulations in a network including one BS, one user, 
and a UAV equipped with IRS. Unless otherwise stated, the simulation parameters are set as $M=64, N_{B}=16, H=100$ $\mathrm{m}, T=25 \mathrm{~s}$ and $V_{m}=25 \mathrm{~m} / \mathrm{s}$. In addition, the transmit power is $20 \mathrm{dBm}$. First, Fig. 2(a) shows the trajectories of UAV under two different network topologies, where the two location sets of BS and user are marked in red and blue, respectively. The initial trajectory of UAV before optimization is a straight line between the given initial and final locations. It can be observed that the UAV flies from the initial location to BS 1, and then changes direction and reaches the final location in the first topology. While for the second topology, the flight of UAV consists of three segments. First, the UAV flies closer to User 2 , then changes its course to BS 2, and finally flies towards the end location. Furthermore, to investigate the impact of the system parameters, we plot the average achievable rate versus the number of IRS elements $M$ in Fig. 2(b) with different number of transmit antennas and time slots. We can see that the average achievable rate is the lowest without reflecting elements, and becomes higher with larger $M$. The average achievable rate also increases with the number of antennas $N_{B}$ at the BS. The reason for the results is that more resources can be leveraged for the active and passive beamforming optimization to achieve better performance. In addition, the rate of $N=40$ outperforms that of $N=25$ and the gap between them enlarges with $M$, due to the fact that the IRS-UAV has more time to help transmit signals in favorable locations. Thus, deploying more antennas at the BS, using more reflecting elements at IRS, and increasing the flying period of UAV are all beneficial to improve the rate performance of the IRS-UAV networks.

\section{IRS-ASSISTED UAV-GROUND WIRELESS NETWORKS}

Although UAVs are likely to establish LoS links in most cases, they still face the challenge of blockages caused by obstacles. In addition, UAV-ground communications may be exposed to severe interference and malicious eavesdropping. To address these issues, IRS can be employed to strengthen the desirable signals at the intended users and overcome blockages. Moreover, IRS is able to deteriorate the unfavorable signals at the unintended users to mitigate the interference and information leakage. In general, there is a trade-off in controlling the phase shifts collaboratively to achieve the two goals at the same time. In the following, we will show how the IRS can be exploited in UAV wireless networks to enhance desired signals and suppress undesired signals simultaneously. Fig. 3 illustrates an IRS-assisted UAV network in the presence of an eavesdropper as a study case to validate the potentials of IRS to improve the security for UAV-ground networks.

\section{A. Transmission Design for IRS-Assisted UAV Networks}

Consider an IRS-assisted UAV network as shown in Fig. 3, where the IRS is placed on the facade of a building to help reflect the signal from the UAV to a ground user. However, there exists a malicious eavesdropper on the ground who intends to wiretap the legitimate information. In contrast with the single-antenna UAV in [12], we equip $N_{u}$ antennas at the UAV to perform the transmit beamforming jointly with the

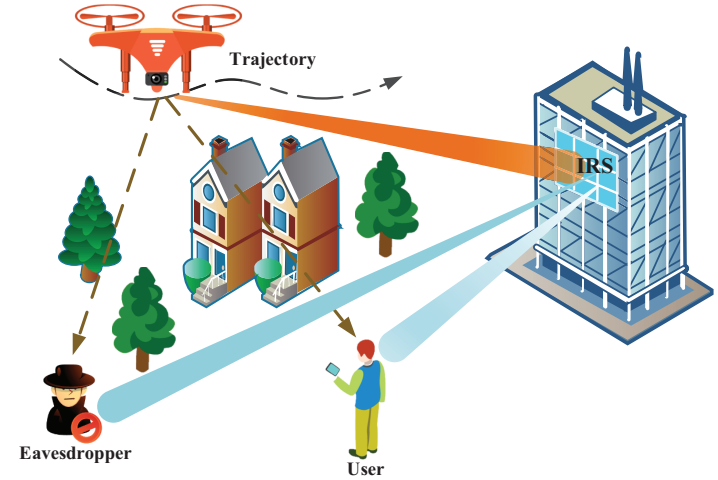

Fig. 3. An IRS-assisted UAV network exposed to eavesdropping.

trajectory and passive beamforming design. Both the user and the eavesdropper have a single antenna. Similar to the system assumption in the previous section, the UAV flies above the area at altitude $H$ with practical mobility constraints, and the trajectory is indicated by the locations within $N$ time slots. The direct links from the UAV to ground nodes are blocked by terrestrial obstacles, and thus, the channels from the UAV to the legitimate user as well as to the eavesdropper are assumed to follow Rayleigh fading. Due to the vertical heights of UAV and IRS, the UAV-to- IRS communication link can be modeled as a LoS channel. For the channels between IRS and ground nodes, we adopt the Rician fading model with both LoS and non-line-of-sight (NLoS) links considered.

To further unveil the potentials of combining IRS and $\mathrm{UAV}$, we aim at maximizing the average secrecy rate of the user by jointly optimizing the transmit beamforming of UAV, the phase shift of IRS, and the UAV trajectory. Due to the presence of eavesdropping and coupling of the optimization variables, the joint problem is non-convex and complicated. To address the problem efficiently, we decompose it into three subproblems and develop an iterative algorithm by alternately optimizing one variable with the other two fixed. Specifically, in each iteration, we first obtain the closed-form solution to transmit beamforming with the aim at maximizing the receiving signal power ratio between the user and the eavesdropper. Then, we proceed to the phase-shift optimization subproblem with the unit modulus constraint which belongs to fractional programming. This subproblem is transformed into a parametric programming by introducing an auxiliary parameter, and is further solved by an iterative algorithm. With the optimized active and passive beamforming, the UAV trajectory optimization can be solved by employing the SCA and first-order Taylor expansions to convert the non-convex objective function and constraints into convex ones. Due to the limited transmit power and phase-shift constraints, the secrecy rate objective is bounded and the algorithm can be guaranteed to converge. With the aid of IRS, the received signals from the UAV can be enhanced at the intended user while the quality of eavesdropping links will be degraded. 


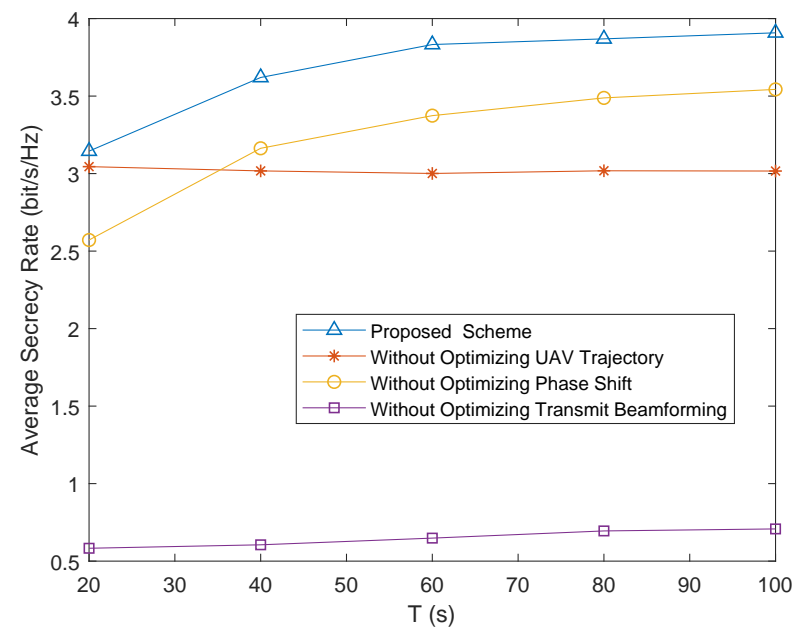

(a)

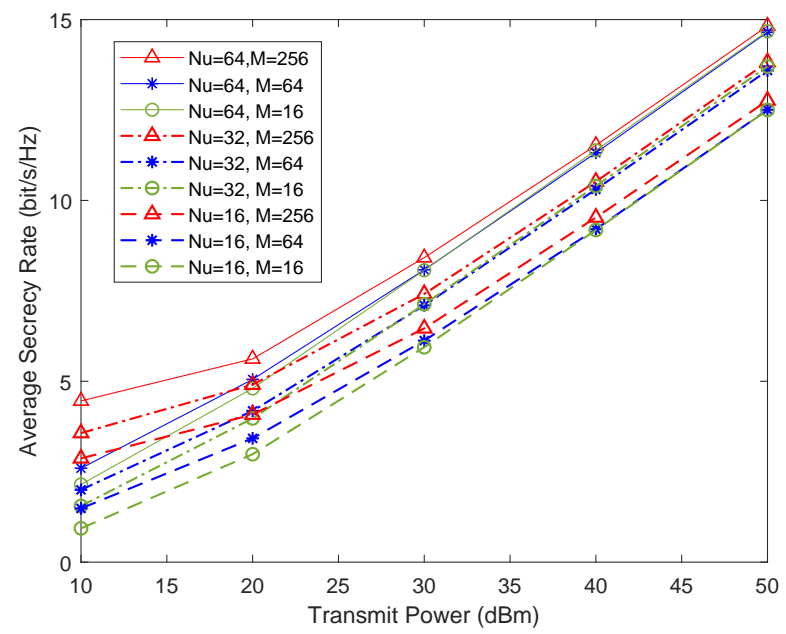

(b)

Fig. 4. (a) The secrecy performance comparison of the proposed scheme and other benchmarks; (b) The secrecy rate versus transmit power with different number of antennas and reflecting elements.

\section{B. Simulation Results}

To verify the superiority of the proposed scheme, the average secrecy rate of different schemes versus flying period $T$ is first compared in Fig. 4(a). We adopt the same default parameters as that in Section III-B. It can be observed that the secrecy rate of the schemes with trajectory optimization all increases with $T$ and finally becomes saturated, due to the fact that the UAV can have more time to serve the users in favorable locations with larger $T$. Conversely, the secrecy rate is nearly a constant without trajectory optimization, because of the uniform mobility of UAV and the average operation on the rate. In addition, the gaps between the proposed scheme and other schemes demonstrate the performance improvement caused by the joint optimization of the transmit beamforming, the phase shift and the UAV trajectory. In Fig. 4(b), we plot the secrecy rate versus the transmit power with different number of antennas and reflecting elements. Obviously, the secrecy rate increases with transmit power. We can also see that when the transmit power is relatively low, increasing the number of antennas or reflecting elements can greatly improve the secrecy rate. However, as the transmit power gets higher, the enhancement from equipping more reflecting elements diminishes compared with that of antennas. Therefore, we should make a trade-off between the number of antennas and that of reflecting elements with the transmit power taken into account in practical scenarios. In the future work, the secure designs with multiple eavesdroppers and the deep reinforcement learning for UAV trajectory will be further investigated.

\section{Open Research Challenges}

Despite the fact that combining UAV and IRS for air-ground networks can provide significant performance improvement, there still exist some challenging issues to be tackled for its design and implementation as follows.

Channel Estimation: To realize the performance enhancement from the joint design of UAV and IRS, accurate CSI estimation is of paramount importance. Imperfect CSI acquisition will degrade the beamforming gain, while higher channel estimation accuracy usually requires higher training overhead and power consumption, especially when the size of IRS is large. Thus, how to improve the accuracy for channel estimation without incurring high overhead in channel training and power consumption is still worth pursuing in the future.

Discrete Phase Shift of IRS: In this article, we consider continuous phase shift of IRS when optimizing the passive beamforming. However, it is impractical due to the fact that implementing high-resolution reflecting elements means higher cost and hardware complexity. Employing the discrete phase shift is more cost-effective, but may result in performance loss compared to the continuous phase shift. Therefore, it is crucial to develop efficient approaches for discrete phase shift with low complexity and performance loss.

Reflection Efficiency: Deploying IRSs in a reflectionefficient way is another vital issue to address their implementations. The location and orientation of IRS are crucial factors to the refection efficiency and thus need to be designed carefully. To compensate for the path loss over a long distance, a largesize IRS with massive elements should be utilized for passive beamforming to enhance the receiving performance, however, with increased cost.

Energy Efficiency: Since no power amplifier is employed, an IRS requires only a limited and static power supply, which is generally neglected. By contrast, energy saving for UAV is urgent due to the limited onboard energy and much higher propulsion energy consumption, which becomes a bottleneck restricting the endurance and performance of UAVs. Thus, energy-efficient designs with the aim at reducing energy expenditure without degrading the performance deserve further investigation for IRS-UAV networks.

Physical Layer Security: In this article, we assume that perfect eavesdropping CSI is available in the legitimate network. However, secure system design at the physical layer with imperfect eavesdropping CSI taken into consideration is more 
robust and practical. Apart from beamforming, cooperative jamming is another effective solution to degrade the qualities of eavesdropping channels. However, UAV can be detected easily by visual or radar scan, which make it a target of jamming attack. Thus, more secure transmission techniques should be collaboratively utilized to enhance the security when combining UAV and IRS in the future.

Environmental Factors: We assume a stable flight of UAV and fixed locations of users in this article. In practice, UAVs usually suffer from inevitable jittering caused by the airflow and vibration, leading to non-negligible channel estimation errors and unstable transmission. As a result, the gain introduced by the joint beamforming design cannot be fully exploited. Then, wind can alter the speed and trajectory of UAV, resulting in safety issues and performance degradation. Furthermore, the QoS of users may not be satisfied due to the dynamic environment and variations of the users' locations.

\section{Conclusions}

The combination of UAV and IRS can help improve the communication quality and provide more flexibility for airground networks. In this article, we discuss the characteristics of UAV and IRS, and present two case studies of combining them to boost the network throughput and ensure secure transmission. First, by mounting the IRS on a mobile UAV, we jointly design the transmit beamforming, the UAV trajectory and the reflecting phase shifts to maximize the average achievable rate. Moreover, the IRS on a building is employed to assist the UAV-ground networks, where the UAV trajectory has been jointly optimized with the active and passive beamforming to maximize the secrecy rate. Last, some open research challenges are discussed on combining UAV and IRS in future air-ground networks.

\section{REFERENCES}

[1] Q. Wu, L. Liu, and R. Zhang, "Fundamental trade-offs in communication and trajectory design for UAV-enabled wireless network," IEEE Wireless Commun., vol. 26, no. 1, pp. 36-44, Feb. 2019.

[2] A. A. Khuwaja, Y. Chen, N. Zhao, M. Alouini, and P. Dobbins, "A survey of channel modeling for UAV communications," IEEE Comm. Surveys Tuts., vol. 20, no. 4, pp. 2804-2821, 2018.

[3] X. Pang, G. Gui, N. Zhao, W. Zhang, Y. Chen, Z. Ding, and F. Adachi, "Uplink precoding optimization for NOMA cellular-connected UAV networks," IEEE Trans. Commun., vol. 68, no. 2, pp. 1271-1283, Feb. 2020.

[4] J. Tang, G. Chen, and J. P. Coon, "Secrecy performance analysis of wireless communications in the presence of UAV jammer and randomly located uav eavesdroppers," IEEE Trans. Inf. Forensics Security, vol. 14, no. 11, pp. 3026-3041, Nov. 2019.

[5] Q. Wu and R. Zhang, "Towards smart and reconfigurable environment: Intelligent reflecting surface aided wireless network," IEEE Commun. Mag., vol. 58, no. 1, pp. 106-112, Jan. 2020.

[6] Q. Wu and R. Zhang, "Intelligent reflecting surface enhanced wireless network via joint active and passive beamforming," IEEE Trans. Wireless Commun., vol. 18, no. 11, pp. 5394-5409, Nov. 2019.

[7] C. Huang, A. Zappone, G. C. Alexandropoulos, M. Debbah, and C. Yuen, "Reconfigurable intelligent surfaces for energy efficiency in wireless communication," IEEE Trans. Wireless Commun., vol. 18, no. 8, pp. 4157-4170, Aug. 2019.

[8] M. Cui, G. Zhang, and R. Zhang, "Secure wireless communication via intelligent reflecting surface," IEEE Wireless Commun. Lett., vol. 8, no. 5, pp. 1410-1414, Oct. 2019.
[9] S. Li, B. Duo, X. Yuan, Y. Liang, and M. Di Renzo, "Reconfigurable intelligent surface assisted UAV communication: Joint trajectory design and passive beamforming," IEEE Wireless Commun. Lett., vol. 9, no. 5, pp. 716-720, May 2020.

[10] L. Ge, P. Dong, H. Zhang, J. Wang, and X. You, "Joint beamforming and trajectory optimization for intelligent reflecting surfaces-assisted UAV communications," IEEE Access, vol. 8, pp. 78702-78712, 2020.

[11] M. Hua, L. Yang, Q. Wu, C. Pan, C. Li, and A. L. Swindlehurst, "UAVassisted intelligent reflecting surface symbiotic radio system." [Online] Available: https://arXiv:2007.14029.

[12] S. Fang, G. Chen, and Y. Li, "Joint optimization for secure intelligent reflecting surface assisted UAV networks," IEEE Wireless Communications Letters, vol. 10, no. 2, pp. 276-280, Feb. 2021.

[13] S. Jiao, F. Fang, X. Zhou, and H. Zhang, "Joint beamforming and phase shift design in downlink UAV networks with IRS-assisted NOMA," $J$. Commun. Inf. Netw., vol. 5, no. 2, pp. 138-149, Jun. 2020.

[14] Q. Zhang, W. Saad, and M. Bennis, "Reflections in the sky: Millimeter wave communication with UAV-carried intelligent reflectors," in Proc. IEEE GLOBECOM'17, pp. 1-6, Waikoloa, HI, USA, Dec. 2019.

[15] Z. Mohamed and S. Aissa, "Leveraging UAVs with intelligent reflecting surfaces for energy-efficient communications with cell-edge users," in Proc. IEEE 2020 ICC Workshops, pp. 1-6, Dublin, Ireland, Jun. 2020.

\section{BIOGRAPHIES}

Xiaowei Pang [GS] (xiaoweipang00@mail.dlut.edu.cn) received the B.S. degree in communication engineering from Northwestern Polytechnical University, Xi'an, China, in 2018. She is currently working toward the $\mathrm{Ph} . \mathrm{D}$. degree in the School of Information and Communication Engineering, Dalian University of Technology, Dalian, China. Her current research interests include UAV, NOMA, IRS, and optimization of wireless networks.

Min Sheng [SM] (msheng@mail.xidian.edu.cn) is a full professor with the State Key Laboratory of ISN, Xidian University. Her research interests include intelligent networks, self-organizing networks, and satellite networks. She was awarded the Distinguished Young Researcher from NSFC, Changjiang Scholar from Ministry of Education, China, elected in "Ten Thousand Talents Program" in 2019, and honored with the Second Prize for the State Technological Innovation Award in 2014 and 2017.

Nan Zhao [SM] (zhaonan@dlut.edu.cn) is a Professor at Dalian University of Technology, China. He received the $\mathrm{Ph} . \mathrm{D}$. degree in information and communication engineering in 2011, from Harbin Institute of Technology, Harbin, China. He received the IEEE Communications Society Asia Pacific Board Outstanding Young Researcher Award in 2018. He is an Editor for IEEE Wireless Communications (magazine), IEEE Transactions on Green Communications and Networking and IEEE Wireless Communications Letters.

Jie Tang [SM] (eejtang@scut.edu.cn) received the B.Eng. degree in Information Engineering from the South China University of Technology, Guangzhou, China, in 2008, the M.Sc. degree in Communication Systems and Signal Processing from the University of Bristol, UK, in 2009, and the Ph.D. degree from Loughborough University, Leicestershire, UK, in 2012. From 2013 to 2015, he was a research associate at the School of Electrical and Electronic Engineering, University of Manchester, UK. He is currently a full professor at the School of Electronic and Information 
Engineering, South China University of Technology, China. $\mathrm{He}$ is currently serving as an Editor for IEEE Systems Journal and IEEE Wireless Communications Letters.

Dusit Niyato [F] (dniyato@ntu.edu.sg) is currently a professor in the School of Computer Science and Engineering, at Nanyang Technological University, Singapore. He received B.Eng. from King Mongkuts Institute of Technology Ladkrabang (KMITL), Thailand in 1999 and Ph.D. in Electrical and Computer Engineering from the University of Manitoba, Canada in 2008. His research interests are in the areas of Internet of Things (IoT), machine learning, and incentive mechanism design.

Kai-Kit Wong [F] (kai-kit.wong@ucl.ac.uk) received the BEng, the MPhil, and the PhD degrees, all in Electrical and Electronic Engineering, from the Hong Kong University of Science and Technology, Hong Kong, in 1996, 1998, and 2001, respectively. After graduation, he took up academic and research positions at the University of Hong Kong, Lucent Technologies, Bell-Labs, Holmdel, the Smart Antennas Research Group of Stanford University, and the University of Hull, UK. He is Chair in Wireless Communications at the Department of Electronic and Electrical Engineering, University College London, UK. His current research centers around $5 \mathrm{G}$ and beyond mobile communications. He is a corecipient of the 2013 IEEE Signal Processing Letters Best Paper Award and the 2000 IEEE VTS Japan Chapter Award at the IEEE Vehicular Technology Conference in Japan in 2000, and a few other international best paper awards. He is Fellow of IEEE and IET and is also on the editorial board of several international journals. He is the Editor-in-Chief for IEEE Wireless Communications Letters since 2020. 